\title{
Scanning training in neurological vision loss: case studies
}

This article was published in the following Dove Press journal:

Eye and Brain

24 May 2010

Number of times this article has been viewed

\author{
Paul Koons' \\ Scott Johnson ${ }^{2}$ \\ John Kingston ${ }^{2}$ \\ Gregory L Goodrich ${ }^{3}$
}

'Blind Rehabilitation Outpatient Specialist, Polytrauma Transitional Rehabilitation Program, Hunter Holmes McGuire Veterans Affairs Medical Center, Richmond,VA, USA; ${ }^{2}$ Western Blind Rehabilitation Center, VA Palo Alto Health Care System, Palo Alto, CA, USA; ${ }^{3}$ Optometric Research Fellowship Program, Psychology Service, VA Palo Alto Health Care System, Palo Alto, CA, USA
Correspondence: Gregory L Goodrich WBRC (I24),VA Palo Alto Health Care System, 380I Miranda Avenue, Palo Alto, CA 94304, USA

Tel +l 6504935000 ext 64385

Email gregory.goodrich@va.gov

\begin{abstract}
The purpose of this paper is to describe the response of a Department of Veterans Affairs medical center's development of a rehabilitation program for patients with hemianopsia. Hemianopsia affects significant numbers of troops returning from Afghanistan and Iraq and their neurological vision loss presented unique challenges in developing an appropriate and effective rehabilitation program. A literature review indicated that existing therapies lacked supporting scientific evidence and that traumatic brain injury (TBI)-related vision loss affects large numbers of civilians. The increasing number of patients with TBI-related vision loss necessitated the development of an innovative program which combined elements of therapies that the literature suggested were most promising. In this paper we briefly review the literature, describe the rehabilitation program developed, and present case studies of two patients who incurred vision loss as a result of a motor vehicle accident and a gunshot wound. The intent of the article is to begin the documentation of our ongoing, evidence-based neurological vision loss rehabilitation program. We also encourage others who do not currently do so to assess the need for implementing vision rehabilitation programs for patients with TBI-related vision loss.
\end{abstract}

Keywords: neurological vision loss, comprehensive neurological vision rehabilitation (CNVR)

\section{Introduction}

Hemianopsia and other field losses often occur with an acquired brain injury $(\mathrm{ABI})^{1,2}$ and recent studies of troops injured while deployed in Iraq and Afghanistan have highlighted the clinical and research importance of these visual deficits. ${ }^{3-5}$ Unfortunately, many severe field losses associated with brain injury go undetected. ${ }^{6}$ The reasons for this have not been examined in depth; however the heterogeneity of the population makes large scale, controlled studies difficult. In brain injury rehabilitation settings clinicians often emphasize visual acuity, which may be within the normal range in cases of hemianopsia (particularly with macular splitting or sparing), and comprehensive visual fields may not be routinely performed. A recent study by Rowe and colleagues ${ }^{7}$ reports that by using a standardized screening $92 \%$ of patients referred for an eye examination were found to have a visual impairment of some form. Our clinical experience suggests that confrontation fields, one of the most commonly used field assessments in brain injury centers, is not a reliable predictor of field loss. Indeed we have had patients who have no visual field loss indicated on their medical records after confrontation testing, present with a hemianopsia when tested with a Goldmann or Humphrey perimeter. Thus referrals in suspected cases of visual impairment following stroke appears to be warranted. Given that referrals 
for vision examinations are not a widely applied standard of care ${ }^{7}$ it is not surprising that the prevalence of visual field loss following ABI has not been well established, although some estimates place the rate as high as $40 \%{ }^{8}$

Hemianopsia is one of the most common disorders following brain injury ${ }^{7,9}$ affecting mobility, reading, driving, and activities of daily life, presenting patients and caregivers with significant challenges. Successful rehabilitation reportedly improves subjective quality of life, and restoration of the ability to perform activities of daily living including educational and occupational tasks, and may, in appropriate cases, allow the individual to drive. ${ }^{10,11}$ Given the level of deficit created by hemianopsia and the value of therapy there has been considerable clinical interest in developing effective rehabilitative strategies. ${ }^{12,13}$

No single rehabilitative technique for visual field loss following an ABI has achieved widespread acceptance due, in large part, to a lack of controlled research ${ }^{14,15}$ and the heterogeneity of the population which makes such studies difficult. Still, a variety of techniques have been proposed including prisms, vision restoration therapy, and scanning therapy, and we will briefly review these.

Prisms are used to relocate the visual image from the lost visual field to the intact field. Perhaps the first use of prisms to treat hemianopsia dates to Young's work in $1929 .{ }^{16}$ In the intervening years prisms have progressed to improved uses of Fresnel lenses ${ }^{11,17-20}$ and to more sophisticated prism placements. ${ }^{19,21}$ While prisms have been found to be helpful to some patients the success rate in the multicenter trial conducted by Bowers, et al ranged from $27 \%$ to $81 \%$ when used to improve general mobility. Studies that have reported success in patient utilization of prisms also report that some patients discontinue their use after a period of time ${ }^{22}$ and the reasons for discontinuation warrant further study.

While prisms relocate the visual image to intact areas of the retina, vision restoration therapy (VRT) seeks to decrease the size of the hemianopic area by capitalizing on brain plasticity to restore vision on the border of the hemianopic field. ${ }^{23}$ VRT is implemented on a computer viewed by the patient using a head-mount to minimize head motion (www. novavision.com). A fixation spot is used to minimize eye movements and stimuli are presented in both the seeing and nonseeing fields. Training is done in the home during two 30-minute sessions six days per week over a 3 to 6 month time period. As patients progress through the VRT sequence the training program is appropriately modified to match the current abilities of the patient. Improvements have been noted as decreases in the border of the hemianopic field of
5 degrees on average, with a range of 0 to 20 degrees, ${ }^{23-26}$ although not all studies have found improvement. ${ }^{27}$

Scanning therapy (ST) seeks to improve visual function in hemianopsia by teaching eye and head scans into the hemianopic field. Scanning with eye movements have been reported to improve visual $\operatorname{search}^{28}$ with scanning improvements of 35 degrees into the hemianopic field. ${ }^{29-31}$ Training strategies generally focus on reading and may include eye movements, head movement, and the addition of other sensory cues including auditory cues and vibratory stimulation of the neck muscles. ${ }^{32,33}$ Rehabilitation of reading performance in hemianopic dyslexia has been shown to be independent of text material with Arabic numerals as effective as words in improving reading performance. ${ }^{34}$ Reading performance in hemianopic alexia has been shown to improve with small-field optokinetic therapy compared to a control population. ${ }^{35,36}$ Verlander and colleagues are an exception in that they focused on mobility performance rather than near task performance. This technique employs a light bar to provide initial assessment and training of head and eye scanning with the training protocol transferred to mobility in graduated stages of difficulty. ${ }^{37}$

Recent reviews of the literature indicate that none of the current training methods reviewed here (prism, vision restitution therapy, and scanning training) are well supported by controlled studies, ${ }^{14,38}$ although some results are encouraging. For example, recent controlled studies by Spitzyna, et $\mathrm{al}^{35}$ and Schuett, et $\mathrm{al}^{34,36}$ support the effectiveness of therapies in improving near tasks (reading). Few studies have examined therapies to improve the patient's mobility in their environment. This leaves clinicians with a dilemma as to how to address the visual deficits in hemianopic patients which impair their ability to move freely and safely within the environment. The current literature does provide some support for scanning training as a promising approach, ${ }^{14,15,39,40}$ although controlled studies comparing the effectiveness of these methods are needed.

\section{Development of the comprehensive neurological vision rehabilitation (CNVR) program}

In 2004 we noted that severely-injured (polytrauma) troops who served in Afghanistan and Iraq were being admitted to our medical center, as well as patients with traumatic brain injury due to motor vehicle accidents, stroke, falls, and other causes. As we reviewed patient outcomes several important factors became readily apparent. For example, for those troops whose vision loss was related to a brain injury 
conventional vision rehabilitation techniques were less successful than desired. To help understand this we developed a comprehensive vision examination protocol designed to assess visual function in patients with varying severities of brain injury. ${ }^{3,41}$ The findings of these studies confirmed that the functional vision losses exhibited by these troops were similar to civilian patients who had suffered brain injuries from stroke, motor vehicle accident, or other causes, although the most common mechanism of injury for troops was a blast event usually from an improvised explosive device, grenade, or mortar shell. ${ }^{4,42}$ The specific nature of the functional losses were diverse, however field loss, including hemianopsia with or without neglect, was relatively common (eg, $\sim 30 \%$ ) and particularly problematic from a treatment point of view.

The VA Palo Alto Medical Center is a unique setting for the treatment of vision loss due to brain injury in that the Center includes both polytrauma and vision rehabilitation services. These facilities allow an unusual degree of multidisciplinary care including optometry, ophthalmology, occupational and physical therapy, neuropsychology, vision rehabilitation, audiology/speech pathology, and other disciplines as needed by the patient. In structuring our program we therefore sought treatment modalities which could benefit from the diversity of resources available. A central therapy selected for the CNVR program was scanning therapy based upon a technique first described by Verlander ${ }^{37}$ and which has recently become commercially available as the Neuro Vision Technology (NVT) System (www.neurovisiontech.com. au). NVT offers education programs in addition to the NVT scanner and training in its use. The NVT scanner consists of a dedicated computer, software program, and the NVT Scanning Device which is a light bar consisting of two rows of 10 colored lights (See Figure 1). The laptop computer provides control of the light presentations and includes assessment protocols, training protocols, and data recording and report generation facilities. An attractive aspect of the NVT, given the available resources and patient needs, is that the therapy protocol transfers from a static training to dynamic mobility situations. In addition to its emphasis on mobility the NVT Systems program was viewed as unique because of the education program offered for vision rehabilitation specialists. The specialists have expertise in age-related vision loss, but lacked the educational and clinical experience in working with ABI patients. The NVT education program was viewed as necessary to equip staff to work effectively with this patient population.

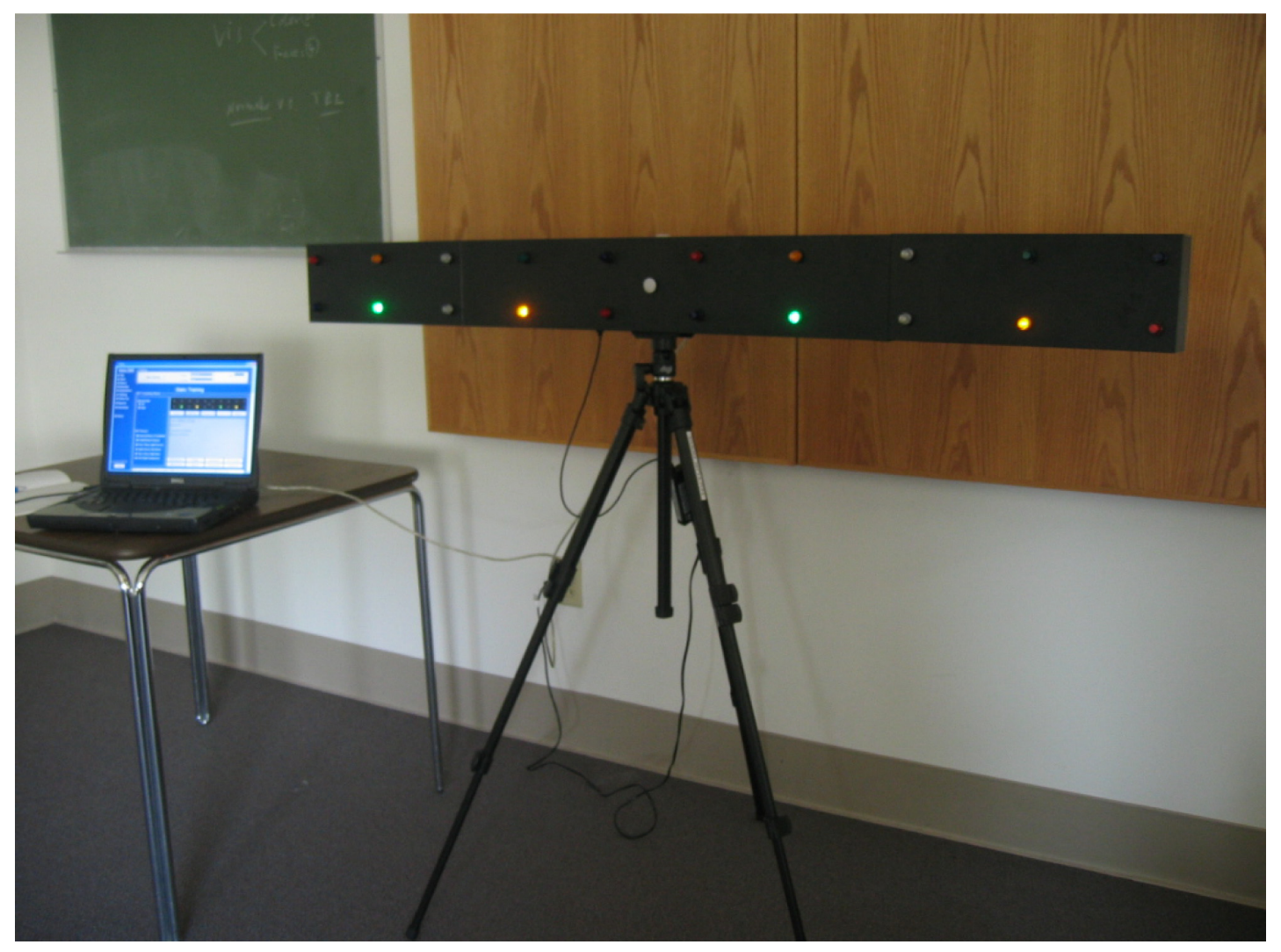

Figure I The NVT Scanning device and laptop computer. 
Initially patients receive a comprehensive functional vision examination by optometry staff experienced in working with brain-injured patients. In patients with hemianopsia, prisms may be prescribed either to improve vision prior to NVT training or as a treatment modality. When indicated by the vision examination, referral for NVT training is made. Initial NVT assessments are completed by either OT or blind rehabilitation specialists who have been certified for the NVT system. Once the initial static assessment and training has been completed the training transitions to a simple indoor mobility route (eg, a quiet hallway) and gradually increases in difficulty to more complex indoor routes (eg, more cross corridors and foot traffic). As the patient's functional capacity improves the training transitions to outdoor environments and eventually into stores, street crossings, and other areas that will be traveled by the patient as part of their daily activities. The ability to transition the specific training from therapy setting to 'real world' environments was a considered a critical factor in the decision to adopt the NVT system. Outcomes, to assess whether there has been an improvement in the patient's visual functioning and mobility, can be ascertained from reviewing the NVT standardized assessment at the commencement of the program and repeating the assessment at the completion of the training.

The following describes two cases. While the apparent clinical success of these has been encouraging, more formal evidence of the system's effectiveness is needed and a controlled study of the NVT and other therapy programs is currently underway.

\section{Case study:AS}

AS was a 22 year old female, active duty Air Force Captain based in Italy who experienced a traumatic brain injury (TBI) as a result of a rollover motor vehicle accident in June 2006. She was initially treated at a local hospital and then transferred to the National Naval Medical Center, Bethesda, Maryland. AS was then transferred to the Department of Veterans Affairs Palo Alto Health Care System for TBI care and acute/postacute rehabilitation. Prior to her injury, she describes herself as very active, with high energy and fairly normal vision, with just 'a lazy left eye' \{strabismic amblyopia (left eye) with best corrected acuities of 20/60 and 20/25 right and left eyes respectively\}. Injuries from the motor vehicle accident included biparietal, bitemporal, and right frontal, with post occipital craniectomy. In conjunction with AS the rehabilitation team developed an initial goal for AS which was to live independently with supportive care from her family.
The initial optometric examination found her visual acuities were 20/60 right eye and 20/400 left eye with left hemiplegia and left neglect \{see Figure 2 (a) and (b) \}. Imaging showed bilateral parietal and temporal contusions, right frontal contusion, and post-occipital injury. A CT scan showed hypodensities in the bilateral frontal and occipital lobes, and 'patchy' hyperdensities in the bilateral parietal loves. Psychological testing found deficits in short term memory, motor planning, visual memory, working memory, and reasoning skills.

Her presenting complaints to the optometrist were that her vision made her feel off-balance and overall things looked blurry. She reported being startled by objects suddenly 'appearing' and lack of visual information on her left side. Her primary complaint was of feeling 'slower' in her processing of information. Her initial assessment noted a left hemiplegia, left neglect, and Rancho IV-V. The initial assessment also determined that AS had impaired mobility due to both her physical and visual status. She was initially wheelchair bound and could transfer with moderate assistance. Within three months she progressed to ambulating with a support cane. NVT training began while AS used a wheelchair and progressed as her mobility improved.

Functional visual assessments indicated:

- Left visual neglect (with no evidence of left side body neglect), as shown by missing the left side of all printed columns, even with strong cueing from her therapist.

- Difficulty with orientation, path finding and verbalizing basic routes on ward.

- Right/left laterality issues and poor ability to verbalize reverse route just traveled.

- Reduced reasoning skills, visual-spatial and visual memory issues, and lack of insight into her deficits.

A multidisciplinary treatment plan was developed including: physical therapy for mobility, strengthening, balance and endurance; occupational therapy for safety, activities of daily living, and range of motion; speech therapy for aphasia; and vision rehabilitation for visual field loss, left visual neglect, and orientation and mobility needs.

AS was one of the first patients to undergo the NVT assessment and training of skills for activities of daily living and mobility. AS's NVT scanning training began with the patient seated 16-20 inches in front of the device and consisted of programmed exercises designed to improve scanning with improved head turning and eye saccades, visual processing and left/right side awareness. Often occupational therapy and physical therapy (OT/PT therapies) would co-treat to monitor AS's standing balance and range of motion while 

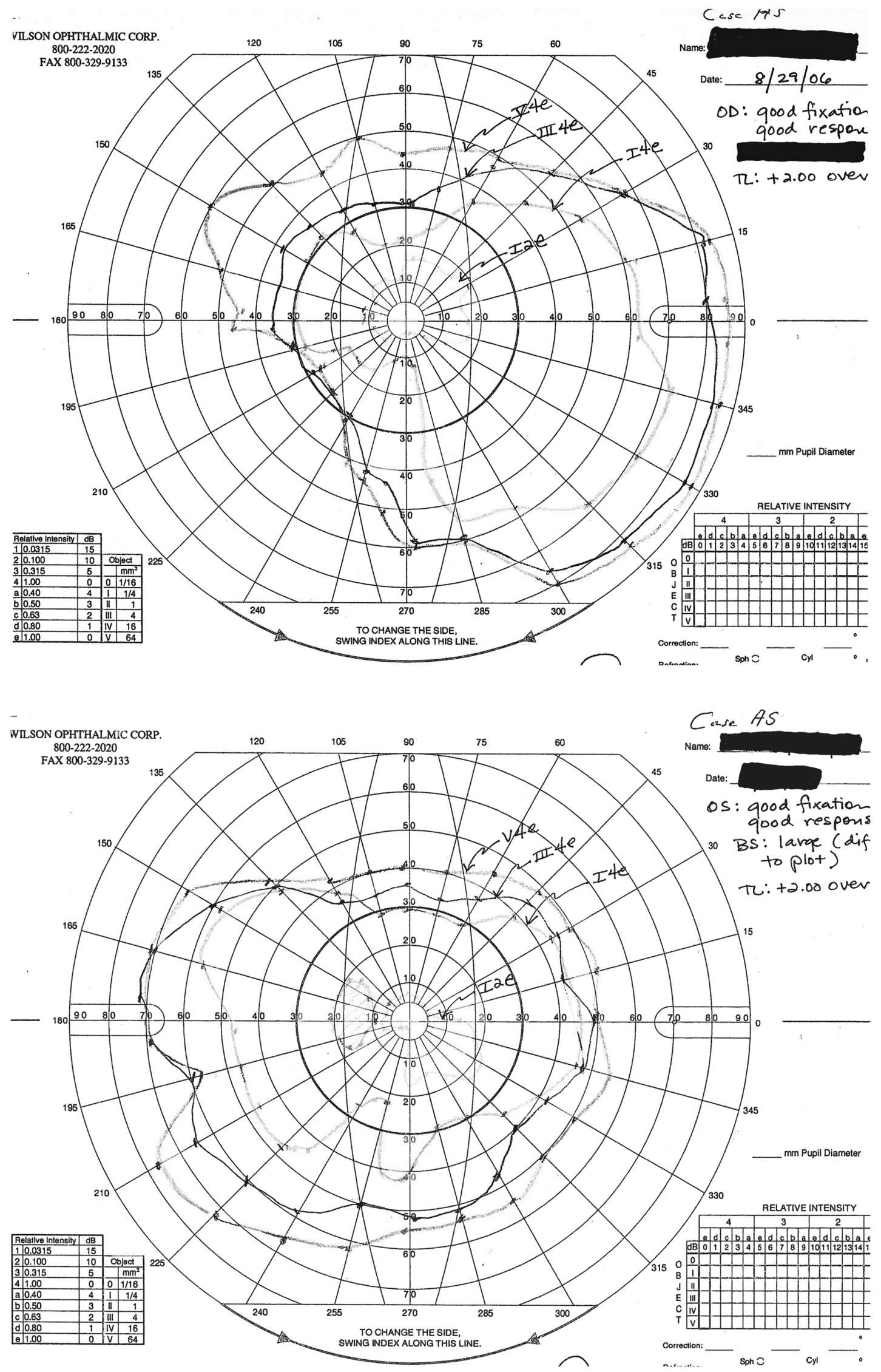

Figure 2 (a) and (b) Right and left visual fields for Case AS. 
she performed static and dynamic scanning training. Next, AS would apply skills into a series of environments ranging from quiet indoor hallways to busy outdoor environments and street crossings. Instructor cues lessened as AS progressed through therapy. AS was extremely motivated and there was a strong carryover of skills learned in NVT training to other therapies including OT, PT, and independence in daily living activities. Inpatient and outpatient therapy lasted 28 weeks broken down by 10 weeks inpatient therapy and 18 weeks outpatient therapy.

Specific steps in the NVT training program included:

- Patient worked up to full scanning in static training (NVT Scanning Device) while seated and standing, commencing with shorter sessions and increasing attention span.

- NVT training included static visual scanning exercises designed to pattern scanning abilities to patient's left side due to visual neglect, increase speed of visual scanning, training for visual-spatial deficits, increase visual memory, visual processing, and gradually increase attention to multiple visual stimuli.

- Transfer of skills to pen and paper tasks, improved column and reading scanning, and increased speed and accuracy of reading and comprehension.

- The NVT device was often used at the beginning of each training session to reinforce degree of head/eye scan, and then apply skills to various environments and pen and paper tasks.

- Dynamic NVT training included orientation to room and ward, path finding to all therapies, target location in quiet and busy hallways, then progressed outdoors to sidewalk route travel, residential travel, supermarket, street analysis and crossing strategies and light business areas.

- As each environment and mobility aid changed eg, wheelchair to walking frame to support cane, scanning was reinforced to ensure gait and balance of the patient was not compromised.

\section{AS outcomes}

Following the acute (10-week) phase of her therapy she had significantly improved saccadic ability (90\%) as measured by performance on the Developmental Eye Movement Test (DEM) and testing of large and micro saccadic eye movement using two colored wands. However her paper and pencil evaluations showed residual deficits in scanning with the patient attaining only about a $25 \%$ success rate in identifying items on tasks such as letter cancellation. In mobility she continued to frequently bump into door frames and other objects in her left visual field.
Following outpatient therapy AS improved markedly in paper and pencil tests and was able to read without losing her place with consistently good reading speed and comprehension. In static tests using the NVT or chalkboard she was able to use eye and head scans to locate objects in her left visual field. Her greater scanning ability transferred to improve her mobility and she only infrequently bumped into door frames or other objects. By completion of outpatient mobility she traveled independently using a support cane and traveled using public buses to get from her apartment to outpatient appointments.

During her rehabilitation she took two driving evaluations to regain a driver's license. She did not pass either evaluation due to her proprioceptive deficits, impaired multi-tasking abilities, slowed reaction times, and problem solving skills. However, post-discharge AS received private driving lessons and passed the state driving test. AS stated she drives on a daily basis but 'things are different now', for example, she drives on well-known routes and avoids unfamiliar routes. She stated that when driving she tries to lessen distractions and consistently manage multi-tasking. She stated her vision scanning became 'automatic' due to her NVT training program. AS's initial treatment goal was to live independently with support from family, however follow-up contact indicates that she now lives independently and thus has exceeded the initial goal.

\section{Case study two RT: Presenting the patient's subjective response to therapy}

The following describes a unique case of rehabilitation provided 55 years post-injury. It is included here as an interesting subjective report both because of the duration between injury and rehabilitation and the insight provided by the patient and therapist.

During the Korean conflict RT sustained a combat injury of a bullet wound which entered the right occipital region and exited the left occipital lobe. Initially he was totally blind and had to crawl down a hill without vision and with severely impaired hearing to escape the enemy. He was successful in contacting American troops who transferred him to a medical unit. From there he was evacuated to the Army Neurological Center at Fort Gambel, Kentucky. He was then sent to Pennsylvania for rehabilitation lasting three months, although he reported that no vision-specific therapy was provided. Following discharge, RT resumed studies at the University of Arizona where he completed his business degree and started law school. He dropped out of law school 
because he could not manage the volume of reading required. He stated there were numerous times in which he recognized that he had not seen something to his left; including people, landmarks, doorways, signs, and words when reading. As is true in many TBI cases RT did not relate these problems to his vision loss. Interestingly, in spite these difficulties he led an active life including being an ocean yacht sailor and a skydiver. RT's realization that his functional problems related to his vision came nearly 55 years after his TBI when an examination revealed that he was legally blind. At that time rehabilitation was prescribed and RT applied for services in our program.

The initial visual examination found visual acuities of $8 / 10$ and $8 / 12$ in the right and left eyes respectively. The functional vision assessment confirmed that he had difficulties with dynamic visual functioning which included: difficulty with field loss due to inconsistent scanning pattern; moving faster than his limited vision could safely monitor; lack of awareness of visual scanning concepts or techniques; running into people or objects, seeing only one person and missing the other people in a group; and inability to see a bus or vehicle that was in his left visual field. TR reported that at some point in the past he had been fitted with prism glasses by a private clinician, but did not find these useful. When fitted he did not receive mobility training specific to prism use. He also did not use a long cane for mobility.

During his three week stay at the Western Blind Rehabilitation Center (WBRC) RT received an intensive assessment and training program with the NVT system and mobility training. RT did not have any cognitive deficit and thus was able to move through the NVT static training quickly and to transfer those skills consistently and accurately to dynamic environments. RT expressed that this was the first time he really understood his visual field and visual perceptual limitations and the implications of these deficits for his everyday functioning. Upon completion of training he expressed feeling better equipped to deal with daily life and felt more positive about his future relative to his visual functioning effectiveness, although he also noted regret that it had taken so long for him to receive rehabilitation. RT expressed these feelings in the following letter he wrote to the Director, Palo Alto Medical Center and to his U.S. Senator.

\section{RT: the patient's point of view}

'On June 13, 1952, I was shot through the head during Korean War combat. The bullet went through the skull behind the ears, blew off the back of the skull, damaged the occipital lobe, and left me legally blind. I lost $75 \%$ of the visual field in both eyes. After a lengthy stay in an Army hospital I was discharged without the benefit of any rehabilitative therapy for my loss of vision. Fifty-five years later I discovered the Veteran's Administration Western Blind Rehabilitation Center (WBRC) in Palo Alto, California.

I was admitted to the Center on January 10, 2007: The staff and I soon realized that their standard program was geared to helping people suffering from vision loss due to eye pathology, not loss of vision due to traumatic brain injury. Therapy for loss of vision due to eye pathology relies heavily upon magnification devices. A person with vision loss due to traumatic brain injury does not need magnification aides, but does need to be trained to bring those objects, which he may not see, into the undamaged portion of his visual field. Upon realization that the normal curriculum at WBRC was not appropriate for traumatic brain injury vision loss, I undertook the NVT assessment and training program for two weeks. The first week I spent training on the NVT Scanning Device and the second week I transferred those skills to all areas of mobility and daily living.

Even though I was fifty-five years late in receiving any visual therapy, I feel this system was beneficial. I did not realize how much of the world I was missing by not scanning fully and how many times that I must have placed my safety at risk'.

\section{The vision therapist's point of view}

Prior to the implementation of the CNVR program, it would have been the expectation of vision therapy staff that RT should have worked out, over a 50+ year period, the need to scan to the left to ensure he saw the full field of view, especially since RT had no other cognitive deficits and functioned, in most instances, independently. The initial NVT and mobility assessments contradicted this assumption resulting in a realization that there is a need to assess the visual functioning of all patients with TBI-related vision loss, even after long intervals between injury and rehabilitation. This was further reinforced to staff by RT's eloquent observation that 'You don't look at what you don't know is there'.

The following describes RT's therapist's view of vision rehabilitation following a TBI after having worked with RT:

'Over some forty years I have worked with many clients and patients with visual problems caused by brain injury from various etiologies. What they all had in common for me was behaviors different from persons with vision loss and no brain damage; behaviors difficult to understand, anticipate or quantify. I had been trained to work with persons whose visual functioning problems were specific to the eyes 
not the brain. Now, working with younger veterans with traumatic brain injury received in combat as well as stroke and other patients who are typically older, I am finding that the NVT training about brain function, visual and cognitive behavior, and patient assessment and training, provides a tool for a systematic approach to what can be, for the therapist an overwhelmingly complex and enigmatic problem; better understanding of and providing effective therapy for clients with visual perceptual and cognitive problems caused by brain injury'.

\section{Discussion}

Troops returning from combat with traumatic brain injury and related visual impairments and dysfunctions became an impetus for initiating a comprehensive neurological vision rehabilitation program within the Palo Alto Health Care System. The types of vision loss were challenging as vision rehabilitation therapies relating to orientation and mobility are not widely used and lack clear scientific validation. In response we developed a rehabilitation program to improve the safety and efficiency of patient mobility, as well as near tasks. The development and documentation of this program is an on-going process as individual patient needs are complex and require therapists to adapt to their specific needs.

Our clinical experience to date, supported by the presented case studies, suggests the program meets the goal of improving visual scanning in functional situations for patients with hemianopsia. The case studies also suggest that rehabilitation programs can be beneficial for patients with TBI-related vision loss. Indeed, as the current program has matured, an increasing number of patients are being referred. Frequently these are patients who had untreated visual loss following stokes or other injury that occurred many years before entry to our program. In developing the program the need for education about TBI-related vision loss for vision rehabilitation therapists became apparent. This realization led to specific educational training that is felt to be a key component in developing a TBI-related vision loss rehabilitation program within a vision rehabilitation program which previously emphasized therapies for age-related visual impairments.

The case studies presented have encouraged us to continue our program development and research activities. We believe the broadening of rehabilitation services directly addresses the question frequently asked by our patients and their families: 'Why has no one referred me before?' Of course, there is no sufficient answer to this question absent the provision of appropriate referrals and vision rehabilitation services.

\section{Acknowledgments}

We wish to thank our colleagues and patients of the Palo Alto Health Care System for their continuing support and for creating an environment that is open to innovative, collaborative program development.

\section{Disclosure}

Gregory L Goodrich, $\mathrm{Ph} \mathrm{D}$ is a research consultant to Neuro Vision Technologies.

\section{References}

1. Suchoff IB, Kapoor N, Ciuffreda KJ, Rutner D, Han E, Craig S. The frequency of occurrence, types, and characteristics of visual field defects in acquired brain injury: a retrospective analysis. Optometry. 2008;79:259-265.

2. Suchoff IB, Kapoor N, Waxman R, Ference W. The occurrence of ocular and visual dysfunctions in an acquired brain-injured patient sample. $J$ Am Optom Assoc. 1999;70:301-308.

3. Goodrich GL, Kirby J, Cockerham G, Ingalla SP, Lew HL. Visual function in patients of a polytrauma rehabilitation center: a descriptive study. J Rehabil Res Dev. 2007;44:929-936.

4. Brahm KD, Wilgenburg HM, Kirby J, Ingalla S, Chang CY, Goodrich GL. Visual impairment and dysfunction in combat-injured military personnel: a population study. Optom Vis Sci. 2009;86:817-825.

5. Stelmack JA, Frith T, Van Koevering D, Rinne S, Stelmack TR. Visual function in patients followed at a Veterans Affairs polytrauma network site: an electronic medical record review. Optomety. 2009;80:419-424.

6. Gianutsos R. Computerized screening: visual field deficits after brain injury. Journal of Behavioral Optometry. 1991;2:143-150.

7. Rowe G, Brand D, Jackson CA, et al. Visual impairment following stroke: do stroke patients require vision assessment. Age Aging. 2009;38:188-193.

8. Kerkoff G. Neurovisual rehabilitation: recent developments and future directions. J Neurol Neurosurg Psychiatry. 2000;68:691-706.

9. Pambakian A, Currie J, Kennard C. Rehabilitation strategies for patients with homonymous visual field defects. J Neuroophthalmol. 2005;25:136-142.

10. Szlyk JP, Mahler CL, Seiple W, Edward DP, Wilensky JT. Driving performance of glaucoma patients correlates with peripheral visual field loss. J Glaucoma. 2005;14:145-150.

11. Szlyk JP, Seiple W, Stelmack J, McMahon T. Use of prisms for navigation and driving in hemianopic patients. Ophthalmic Physiol Opt. 2005;25:128-135.

12. Pambakian AL, Wooding DS, Patel N, Morland AB, Kennard C, Mannan SK. Scanning the visual world: a study of patients with homonymous hemianopia. J Neurol Neurosurg Psychiatry. 2000;69:751-759.

13. kerkhoff G. Neurovisual rehaiblitation: recent developments and future directions. J Neurol Neurosurg Psychiatry. 2000;68:691-706.

14. Bouwmeester L, Heutink J, Lucas C. The effect of visual training for patients with visual field defects due to brain damage: a systematic review. J Neurol Neurosurg Psychiatry. 2007;78:555-564.

15. Riggs RV, Andrews K, Roberts P, Gilewski M. Visual deficit interventions in adult stroke and brain injury: a systematic review. Am J Phys Med Rehabil. 2007;86:853-860.

16. Young CA. Homonymous hemianopsia during pregnancy aided by reflecting prisms. Arch Ophthalmol. 1929;2:560.

17. Perlin RR, Dziadul J. Fresnel prisms for field enhancement of patients with constricted or hemianopic visual fields. J Am Optom Assoc. 1991;62:58-64.

18. Perez A, Jose R. The use of Fresnel and ophthalmic prisms in the management of patients with hemianopic field visual field loss: a suggested multidisciplinary approach. $J$ Vis Impair Blind. 2003;97:173-176. 
19. Peli E. Treating hemianopia using prisms to create peripheral diplopia. In: Stuen C, Arditi A, Horowitz A, Lang MA, Rosenthal B, Seidman K, editors. Vision '99': Vision Rehabilitation: Assessment, Intervention and Outcomes. New York, NY: Swets \& Zeitlinger Publishers bv; 2000:104-109.

20. Perez AM, Jose RT. Fitting prisms for visual field loss: A clinical case presentation. In: Stuen C, Arditi A, Horowitz A, Lang MA, Rosenthal B, Seidman K, editors. Vision '99': Vision Rehabilitation: Assessment, Intervention and Outcomes. New York, NY: Swets \& Zeitlinger Publishers bv; 2000:10-112.

21. Bowers AR, Keeney K, Peli E. Community-based trial of a peripheral prism visual field expansion device for hemianopia. Arch Ophthalmol. 2008;126:657-664.

22. Giorgi RG, Woods RL, Peli E. Clinical and laboratory evaluation of peripheral prism glasses for hemianopsia. Optom Vis Sci. 2009;86:492-502.

23. Sabel BA, Kasten E. Restoration of vision by training of residual functions. Curr Opin Ophthalmol. 2000;11:430-436.

24. Sabel BA, Kenkel S, Kasten E. Vision restoration therapy (VRT) efficacy as assessed by comparative perimetric analysis and subjective questionnaires. Restor Neurol Neurosci. 2004;22:399-420.

25. Julkunen L, Tenovuo O, Jaaskelainen S, Hamalainen H. Rehabilitation of chronic post-stroke visual field defect with computer-assisted training: a clinical and neurophysiological study. Restor Neurol Neurosci. 2003;21:19-28.

26. Hyvärinen L, Raninen AN, Näsänen RE. Vision rehabilitation in homonymous hemianopia. Neuro ophthalmology. 2002;27:97-102.

27. Reinhard J, Schreiber A, Schiefer U, et al. Does visual restitution training change absolute homonymous visual field defects? A fundus controlled study. Br J Ophthalmol. 2005;89:30-35.

28. Zihl J. Eye movement patterns in hemianopic dyslexia. Brain. 1995;118:891-912.

29. Zihl J. Visual scanning behavior in patients with homonymous hemianopia. Neuropsychologia. 1995;33:287-303.

30. Zihl J. Oculomotor scanning performance in subjects with homonymous visual field disorders. Vis Impair Res. 1999;1:23-31.
31. Hirayama K, Sakai S, Yamawaki R, et al. Visual search training for a case of homonymous field defect with multiple visual dysfunctions. No to Shinkei. 2004;56:403-413.

32. Mascord DJ, Heath RA. Behavioral and physiological indices of fatigue in a visual tracking task. J Safety Res. 1992;23:19-25.

33. Schindler I, Kerkhoff G, Karnath HO, Keller I, Goldenberg G. Neck muscle vibration induces lasting recovery in spatial neglect. $J$ Neurol Neurosurg Psychiatry. 2002;73:412-419.

34. Schuett S, Heywood CA, Kentridge RW, Zihl J. The significance of visual information processing in reading: insights from hemianopic dyslexia. Neuropsychologia. 2008;46:2445-2462.

35. Spitzyna GA, Wise RJ, McDonald SA, et al. Optokinetic therapy improves text reading in patients with hemianopic alexia: a controlled trial. Neurology. 2007;68:1922-1930.

36. Schuett S, Kentridge RW, Zihl J, Heywood CA. Are hemianopic reading and visual exploration impairments visually elicited? New insights from eye movements in simulated hemianopia. Neuropsychologia. 2009;47:733-746

37. Verlander D, Hayes A, McInnes JK, et al. Assessment of clients with visual spatial disorders: a pilot study. Vis Impair Res. 2000;2:129-142.

38. Pelak VS, Dubin M, Whitney E. Homonymous hemianopia: a critical analysis of optical devices, compensatory training, and novavision. Curr Treat Options Neurol. 2007;9:41-47.

39. Roth T, Sokolov AN, Messias A, Roth P, Weller M, Trauzettel-Klosinski S. Comparing explorative saccade and flicker training in hemianopia: A randomized controlled study. Neurology. 2009;72: 324-331.

40. Marshall RS. Rehabilitation approaches to hemineglect. Neurologist. 2009; 15:185-192.

41. Lew HL, Poole J, Vanderploeg R, et al. Program development and defining characteristics of returning military in a VA Polytrauma Network Site. J Rehabil Res Dev. 2007;44:1027-1034.

42. Cockerham GC, Goodrich GL, Bower KS, et al. The eye and visual function in traumatic brain injury. J Rehabil Res Dev. 2009;46:811-818.
Eye and Brain

\section{Publish your work in this journal}

Eye and Brain is an international, peer-reviewed, open access journal focusing on clinical and experimental research in the field of neuro-ophthalmology. All aspects of patient care are addressed within the journal as well as basic research. Papers covering original research, basic science, clinical and epidemiological studies, reviews and evaluations, guidelines, expert opinion and commentary, case reports and extended reports are welcome. The manuscript management system is completely online and includes a very quick and fair peer-review system, which is all easy to use. Visit http://www.dovepress.com/testimonials.php to read real quotes from published authors. 